\title{
Diluted Antiferromagnets in Exchange Bias: Proof of the Domain State Model
}

\author{
P. Miltényi,* M. Gierlings, J. Keller, B. Beschoten, and G. Güntherodt \\ 2.Physikalisches Institut, RWTH Aachen, 52056 Aachen, Germany \\ U. Nowak ${ }^{\dagger}$ and K.D. Usadel \\ Theoretische Tieftemperaturphysik, Gerhard-Mercator-Universität-Duisburg, 47048 Duisburg, Germany \\ (Received 23 December 1999)

\begin{abstract}
The exchange bias coupling at ferromagnetic/antiferromagnetic interfaces in epitaxially grown $\mathrm{Co} / \mathrm{CoO}$ layers can intentionally be increased by a factor of up to 3 if the antiferromagnetic $\mathrm{CoO}$ layer is diluted by nonmagnetic defects in its volume part away from the interface. Monte Carlo simulations of a simple model of a ferromagnetic layer on a diluted antiferromagnet show exchange bias and explain qualitatively its dilution and temperature dependence. These investigations reveal that diluting the antiferromagnet leads to the formation of volume domains, which cause and control exchange bias.
\end{abstract}

PACS numbers: 75.70.Cn, 75.40.Mg, 75.50.Lk, 85.70.-w

Exchange bias results from the exchange coupling at interfaces of ferromagnetic(FM)/antiferromagnetic(AFM) layers leading to a shift of the hysteresis loop along the magnetic field axis. This shift occurs after cooling the system with the magnetized FM layer below the Néel temperature of the AFM or for layer deposition in an external magnetic field. Although this effect has been well known for many years $[1,2]$ and is already intensively exploited in magnetic sensor systems (spin valve [3] and magnetoresistance devices [4]), its microscopic origin is still discussed controversially.

In the approach of Malozemoff [5], exchange bias (EB) is attributed to the formation of domain walls in the AFM below $T_{N}$ perpendicular to the FM/AFM interface due to interface roughness. During cooling these AFM domains lead to a small net magnetization at the FM/AFM interface. This magnetization is then increasingly stabilized toward low temperatures, consequently shifting the hysteresis loop. However, due to the lack of appropriate imaging methods, these domains have never been observed directly. A recent micromagnetic model by Schulthess and Butler [6] yields EB only if uncompensated AFM spins are assumed at the interface, which were observed experimentally [7]. Other EB models [8,9] assume the formation of a domain wall in the AFM parallel to the interface during the hysteresis loop.

In this Letter we show that it is possible to strongly influence (enhance or decrease) $\mathrm{EB}$ in $\mathrm{Co} / \mathrm{CoO}$ bilayers by diluting the antiferromagnetic $\mathrm{CoO}$ layer, i.e., by inserting nonmagnetic substitutions $\left(\mathrm{Co}_{1-x} \mathrm{Mg}_{x} \mathrm{O}\right)$ or defects $\left(\mathrm{Co}_{1-y} \mathrm{O}\right)$ not at the FM/AFM interface but rather throughout the volume part of the AFM layer (see upper left inset of Fig. 2). A 0.4-nm-thick $\mathrm{CoO}$ layer with minimum defect concentration was placed at the interface for all samples investigated. The strong dependence of the EB on the dilution of the AFM layer will be argued to have its origin in the formation of a domain state in the volume part of the AFM. We demonstrate that in our systems EB is primarily due not to disorder or defects at the interface but rather to a domain state in the volume part of the AFM which triggers the spin arrangement and the FM/AFM exchange interaction at the interface. These findings are an important new step in understanding EB, explaining straightforwardly a number of puzzling experimental observations. Our main conclusions are supported by Monte Carlo simulations performed at finite temperatures.

First we will describe the sample preparation by molecular beam epitaxy (MBE) and characterization by reflection high energy electron diffraction (RHEED), low energy electron diffraction (LEED), and atomic force microscopy. We have chosen the AFM CoO for its experimentally convenient Néel temperature $\left(T_{N}=291 \mathrm{~K}\right)$ and because it allows for two different ways to introduce nonmagnetic defects. The first type of defect can be formed with nonmagnetic $\mathrm{MgO}$ since it has the same crystal structure as $\mathrm{CoO}$ and only a $1.1 \%$ lattice mismatch. Second, Co can oxidize in any stoichiometry $\left(\mathrm{Co}_{1-y} \mathrm{O}\right)$ ranging from $\mathrm{CoO}$ to $\mathrm{Co}_{3} \mathrm{O}_{4}(y=0.25)$ [10]. $\mathrm{Co}_{3} \mathrm{O}_{4}$ has a Néel temperature of $T_{N}=33 \mathrm{~K}$. This means that, by Co deficiency or, in other words, overoxidation of $\mathrm{CoO}$, defects can be created in the magnetic structure.

As a substrate for film deposition we used (0001)oriented single crystalline sapphire $\left(\mathrm{Al}_{2} \mathrm{O}_{3}\right)$, which was rinsed in methanol before transfer into the MBE chamber. Prior to film deposition the substrates were heated to $T=775 \mathrm{~K}$ for $1 \mathrm{~h}$ in order to outgas the substrate holder and then cooled to the Co growth temperature of $T=575 \mathrm{~K} .6 \mathrm{~nm}$ of Co were deposited by electron beam evaporation at a rate of $0.2 \mathrm{~nm} / \mathrm{min}$ and annealed at a temperature of $T=775 \mathrm{~K}$ for $10 \mathrm{~min}$. The pressure during evaporation was better than $5 \times 10^{-9}$ mbar. For all samples a 0.4-nm-thick $\mathrm{CoO}$ film was grown at a temperature of $T=350 \mathrm{~K}$ and at a rate of $0.3 \mathrm{~nm} / \mathrm{min}$ by evaporating $\mathrm{Co}$ in an oxygen atmosphere of $3.3 \times 10^{-7}$ mbar. This ensures that all samples have an identical FM/AFM interface independent of the dilution of the following AFM layer. Then for one set of samples a $20 \mathrm{~nm} \mathrm{Co}_{1-x} \mathrm{Mg}_{x} \mathrm{O}$ 
layer was grown by electron beam evaporation of Co and $\mathrm{MgO}$ at a temperature of $T=350 \mathrm{~K}$, a rate of $0.3 \mathrm{~nm} / \mathrm{min}$ and an oxygen pressure of $3.3 \times 10^{-7} \mathrm{mbar}$. The $\mathrm{Mg}$ concentration was varied between $0 \%$ and $100 \%$. For a second set of samples, $20 \mathrm{~nm}$ of $\mathrm{Co}_{1-y} \mathrm{O}$ were grown at a temperature of $T=350 \mathrm{~K}$ and with variable cobalt deficiency $y$ by choosing the oxygen pressure between $3.3 \times 10^{-7}$ mbar and $1.0 \times 10^{-5}$ mbar. All thicknesses of the different layers were controlled by a calibrated microbalance and ex situ atomic force microscopy.

In situ RHEED studies of the Co layer indicate diffraction from a two-dimensional surface. LEED investigations reveal a clear sixfold symmetry indicating that presumably a mixture of hcp (0001) and fcc (111) phases is present due to stacking faults. In Fig. 1, RHEED images are shown for a $\mathrm{Co}_{1-y} \mathrm{O}$ layer grown at an oxygen pressure of (a) $p\left(\mathrm{O}_{2}\right)=3.3 \times 10^{-7} \mathrm{mbar}$ and (b) $p\left(\mathrm{O}_{2}\right)=1.0 \times 10^{-5}$ mbar. For each $\mathrm{Co}_{1-y} \mathrm{O}$ layer, two in-plane orientations denoted as direction $0^{\circ}$ and $30^{\circ}$ of the incident electron beam relative to the sapphire [1 $1 \overline{1} 20]$ axis were chosen (see two vertical panels in Fig. 1). The diffraction patterns from the $\mathrm{Co}_{1-y} \mathrm{O}$ layer show a transmission image, i.e., diffraction from a rough surface with islands [11].

(a)
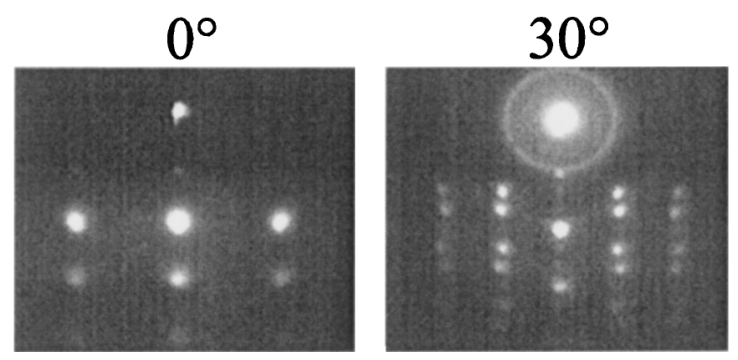

(b)
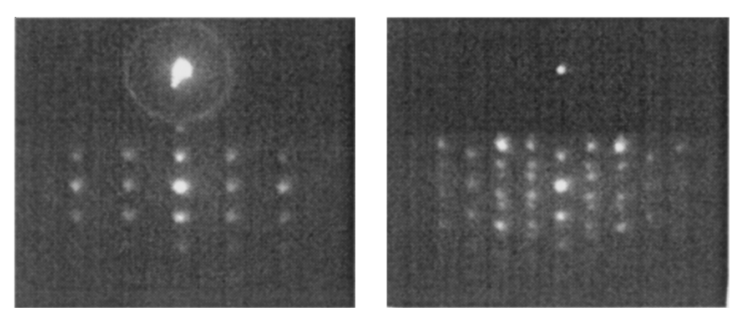

(c)
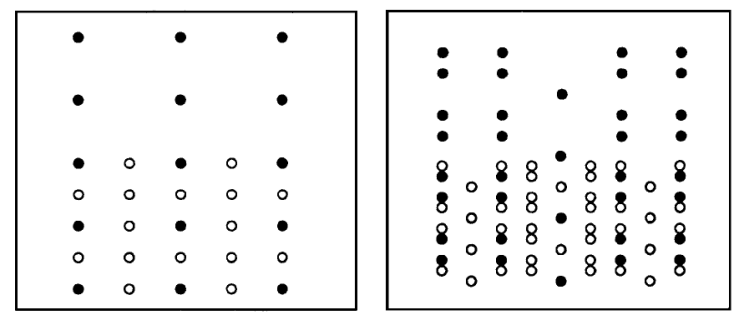

FIG. 1. RHEED images of the $\mathrm{Co}_{1-y} \mathrm{O}$ layer prepared at (a) $p\left(\mathrm{O}_{2}\right)=3.3 \times 10^{-7} \operatorname{mbar}(y \approx 0)$ and (b) $p\left(\mathrm{O}_{2}\right)=$ $1.0 \times 10^{-5}$ mbar. (c) Calculated reflections of the diffraction pattern of $\mathrm{CoO}(111)$ with $60^{\circ}$ twins but only the solid dots fulfill the diffraction condition for an fcc lattice. The two vertical panels show the patterns for $0^{\circ}$ and $30^{\circ}$ in plane orientation of the incident electron beam relative to the sapphire [1120] axis.
In order to explain the observed RHEED patterns, a (111) orientation of the $\mathrm{Co}_{1-y} \mathrm{O}$ layer is assumed, where twins with $60^{\circ}$ in-plane rotation relative to each other are present. The expected diffraction patterns are shown in Fig. 1(c). The solid and open circles are both reciprocal lattice points; however, only the solid circles fulfill the diffraction condition for an fcc lattice. For both directions of $0^{\circ}$ and $30^{\circ}$ the pattern of full circles fits very well to image Fig. 1(a), while, for explaining the image of Fig. 1(b), all reciprocal lattice points have to be taken into account. We conclude that at an oxygen pressure of $p\left(\mathrm{O}_{2}\right)=3.3 \times 10^{-7}$ mbar, practically defect-free $\mathrm{CoO}$ is deposited, while, for $p\left(\mathrm{O}_{2}\right)=1.0 \times 10^{-5} \mathrm{mbar}$, $\mathrm{Co}_{1-y} \mathrm{O}$ is formed. For the $\mathrm{Co}_{1-y} \mathrm{O}$ the destructive interference from the fcc lattice is suppressed because of some empty lattice sites, and more diffraction spots become visible. Without the assumption of twins the symmetrical RHEED patterns in the right panel $\left(30^{\circ}\right)$ of Figs. 1(a) and 1(b) cannot be explained. Atomic force microscope images (not shown) reveal crystallites with a size of approximately $50 \mathrm{~nm}$. This growth mode corresponds to that reported in Ref. [12]. Hence, we conclude that on epitaxial $\mathrm{Co}(111)$ layers grown on sapphire(0001) the $0.4 \mathrm{~nm}$ $\mathrm{CoO}$ and $20 \mathrm{~nm} \mathrm{Co}_{1-y} \mathrm{O}$ or $20 \mathrm{~nm} \mathrm{Co}_{1-x} \mathrm{Mg}_{x} \mathrm{O}$ were deposited with $60^{\circ}$ twins.

The magnetic characterization of the samples was performed with a superconducting quantum interference device (SQUID) magnetometer. The EB field was determined from hysteresis loops measured at temperatures between 5 and $320 \mathrm{~K}$. Figure 2 shows hysteresis loops at $T=5$ and $T=320 \mathrm{~K}$ of the sample with the $\mathrm{Co}_{1-y} \mathrm{O}$ layer grown at $p\left(\mathrm{O}_{2}\right)=3.0 \times 10^{-6}$ mbar. The temperature dependence of the EB field of this sample is shown in the inset at the lower right. The blocking temperature is close to $T_{N}(\mathrm{CoO})=291 \mathrm{~K}$.

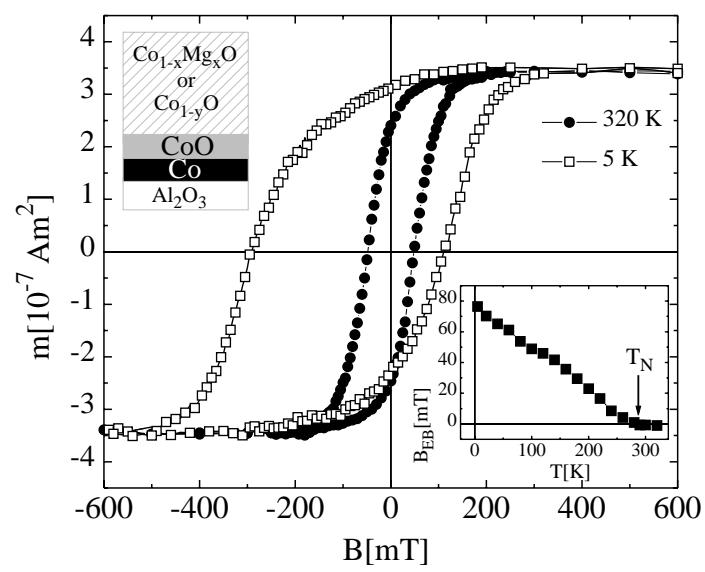

FIG. 2. Hysteresis loops of $\mathrm{Co}_{1-y} \mathrm{O} / \mathrm{CoO} / \mathrm{Co} / \mathrm{Al}_{2} \mathrm{O}_{3}(0001)$ at $T=5$ and $320 \mathrm{~K}$ with the $\mathrm{Co}_{1-y} \mathrm{O}$ prepared at $p\left(\mathrm{O}_{2}\right)=$ $3.0 \times 10^{-6}$ mbar. The lines are a guide to the eye. The upper left inset shows schematically the layer structure of the sample. The lower right inset shows the EB field as a function of temperature. 

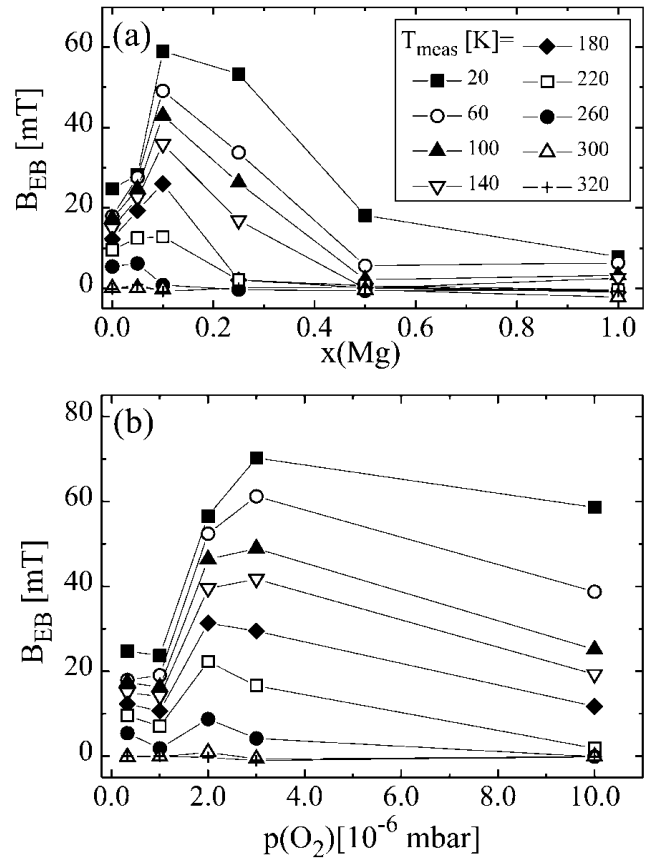

FIG. 3. (a) EB field as function of the Mg concentration $x$ in the $\mathrm{Co}_{1-x} \mathrm{Mg}_{x} \mathrm{O}$ layer for various temperatures. (b) EB field as a function of the oxygen pressure during deposition of the $\mathrm{Co}_{1-y} \mathrm{O}$ layer for various temperatures. Lines are guides to the eye.

In Fig. 3(a) the EB field is shown as a function of the $\mathrm{Mg}$ concentration $x$ for different temperatures. In Fig. 3(b) the EB field is shown at different temperatures as a function of the oxygen pressure $p\left(\mathrm{O}_{2}\right)$ during the preparation of the $\mathrm{Co}_{1-y} \mathrm{O}$ layer. In both cases it can clearly be seen that defects in the AFM layer increase the EB field by a factor of 2-3. At very high dilution the EB starts to drop since the AFM order is destroyed. These results can be explained within the framework of the physics of diluted antiferromagnets.

It is by now well known that a diluted antiferromagnet in an external magnetic field (DAFF) develops a domain state when cooled below its Néel temperature [13]. The driving force for the domain formation is a statistical imbalance of the number of impurities of the two sublattices in a finite region of the DAFF. This leads to a net magnetization which couples to the external field. The necessary energy increase due to the formation of domain walls can be minimized if the domain walls pass preferentially through nonmagnetic defects at no cost of exchange energy [14]. Hence, defects substantially favor the formation of domains in the DAFF. Applying this to the present system the domains in the volume of the AFM layer alter the spin structure at the FM/AFM interface leading to a small net magnetization, which results in EB.

To support this picture we performed Monte Carlo simulations of a model consisting of a FM monolayer (size $128 \times 128$ sites) exchange coupled to a diluted AFM film consisting of 9 layers. The FM layer is described by a clas- sical Heisenberg model (exchange constant $J$ ). The dipolar interaction is approximated by including an anisotropy term (shape anisotropy) leading to an in-plane magnetization. Also, we introduce an easy axis in the FM (anisotropy constant $0.1 J$ ) in order to obtain well-defined hysteresis loops. In view of the rather strong anisotropy in $\mathrm{CoO}$ we assume an Ising Hamiltonian for the DAFF (see Ref. [14] and references therein; exchange constant $J_{\mathrm{AF}}=-J / 2$ ), where the easy axis is parallel to that of the FM. For the coupling between AFM and FM we assume the same coupling constant $\left(J_{\mathrm{INT}}=J_{\mathrm{AF}}\right)$ as for the AFM. In order to model the same interface structure for all calculations the interface monolayer of the AFM was fixed at a dilution of $50 \%$, while we vary the dilution in the volume of the AFM film (8 layers), in analogy to what was done in the experiments.

The system is cooled in zero field from above to below the ordering temperature of the DAFF. While the FM is long-range ordered during the cooling procedure the AFM develops due to its coupling to the FM layer a domain state with a surplus magnetization similar to a DAFF when cooled in an external field. Then hysteresis loops were simulated with the field $B$ almost parallel to the easy axis of the system.

A typical hysteresis loop is shown in Fig. 4(a). Note that the interface magnetization of the AFM is shifted to negative values since the exchange coupling to the FM is negative. This shift of the magnetization of the AFM is responsible for the EB. Figure 4(b) shows the dependence of the $\mathrm{EB}$ on the dilution. The overall qualitative
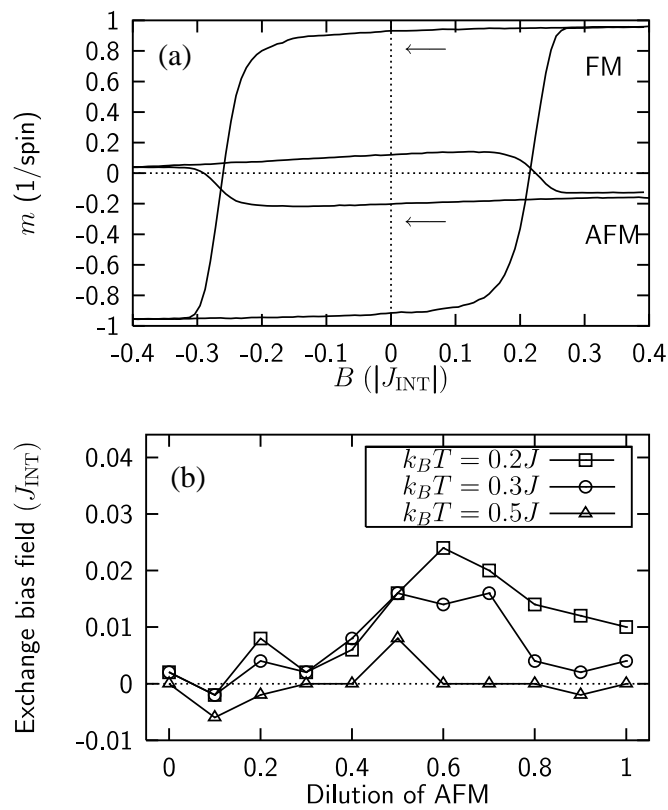

FIG. 4. (a) Simulated hysteresis loop of the model explained in the text at $k_{B} T=0.2 \mathrm{~J}$. Shown is the magnetization per spins of the FM monolayer and of the interface AFM monolayer. (b) EB field as a function of the dilution of the AFM volume for $k_{B} T=0.2 \mathrm{~J}, 0.3 \mathrm{~J}$, and $0.5 \mathrm{~J}$. 
agreement with the experimental finding is clearly given. Especially, we observe a strong increase of the EB with the dilution before it drops again for larger dilution due to the loss of connectivity of the AFM spin lattice. Since the thus appearing isolated spin clusters do not contribute to the AFM domain structure on longer time scales this leads to a decrease of EB for very high dilution. We would like to emphasize that the maximum strength of the simulated EB is of the order of a few percent of the interface coupling constant in agreement with typical measurements [2]. For small dilution our model does not show any EB in contrast to our experimental findings. This can be explained by grain boundaries in the twinned AFM layer which reduce the domain wall energy, thus leading to EB without intentional dilution. On the other hand, in EB systems as, for instance, permalloy on a single crystal of $\mathrm{CoO}$ [15] and $\mathrm{Fe}$ on a single crystal of $\mathrm{FeF}_{2}$ [16] only very small EB fields are found in agreement with our model calculations. For epitaxial NiO layers half the EB field was found in comparison to polycrystalline $\mathrm{NiO}$ layers [17]. Our findings concerning the role of defects in the volume of the AFM layer complement the ideas of Malozemoff [5] who assumed exclusively an interface roughness as the cause of domains in the AFM.

Assuming that the AFM used in EB systems is in a domain state similar to that of a DAFF, further experimental findings can be understood straightforwardly. First, it is the positive EB [18] or reduced EB [19] observed experimentally in strong cooling fields. During the cooling in a strong field the AFM forms domains with the surplus magnetization being parallel to the external field and also to the magnetization of the FM. If the coupling between AFM and FM is negative as assumed in Ref. [18], this will yield positive EB. Second, the time dependence of EB in $\mathrm{Ni}_{66} \mathrm{Co}_{18} \mathrm{Fe}_{16} / \mathrm{NiO}$ and $\mathrm{Ni}_{66} \mathrm{Co}_{18} \mathrm{Fe}_{16} / \mathrm{FeMn} \mathrm{[20]} \mathrm{after} \mathrm{the}$ FM layer is reversed from its cooling field direction can be understood in the framework of the dynamics of the DAFF. Here it is known that the remanent magnetization of the domain state relaxes nonexponentially on extremely long time scales after the field is switched off $[21,22]$. This relaxation of the magnetization of the DAFF is linked directly to the relaxation of the $\mathrm{EB}$, if one assumes that the domains in the AFM are responsible for EB. Third, the reason for the so-called training effect can be understood from Fig. 4(a), where it is shown that the hysteresis loop of the AFM is not closed on the right-hand side. This implies that the magnetization of the AFM is lost partly during the hysteresis loops due to a rearrangement of the AFM domain structure.

In conclusion, we have shown both by experiments and by Monte Carlo simulations that diluting the AFM layer in the volume part away from the FM/AFM interface significantly enhances EB. This dilution induced by nonmagnetic defects supports the formation of volume domains in the AFM which are crucial for the existence of exchange bias.
Since disorder in the AFM layer of exchange bias systems is rather common, our model yields a general understanding of the microscopic origin of exchange bias. Considering the physical properties of diluted antiferromagnets, important features of EB systems can be explained such as the differences in EB using thin film or single crystal antiferromagnets, positive EB as well as temperature and time dependence of EB, and others. Further work following these lines is in progress and will be published elsewhere.

This work has been supported by the Deutsche Forschungsgemeinschaft through SFB 341 and 491.

*Email address: peter.miltenyi@ @physik.rwth-aachen.de

${ }^{\dagger}$ Email address: uli@thp.uni-duisburg.de

[1] W. H. Meiklejohn and C.P. Bean, Phys. Rev. 102, 1413 (1956); 105, 904 (1957).

[2] J. Nogués and Ivan K. Schuller, J. Magn. Magn. Mater. 192, 203 (1999).

[3] B. Dieny et al., Phys. Rev. B 43, 1297 (1991).

[4] C. Tsang, J. Appl. Phys. 55, 2226 (1984).

[5] A. P. Malozemoff, Phys. Rev. B 35, 3679 (1987); J. Appl. Phys. 63, 3874 (1988); Phys. Rev. B 37, 7673 (1988).

[6] T. C. Schulthess and W. H. Butler, Phys. Rev. Lett. 81, 4516 (1998); J. Appl. Phys. 85, 5510 (1999).

[7] K. Takano et al., Phys. Rev. Lett. 79, 1130 (1997); J. Appl. Phys. 83, 6888 (1998).

[8] D. Mauri et al., J. Appl. Phys. 62, 3047 (1987).

[9] M. D. Stiles and R. D. McMichael, Phys. Rev. B 59, 3722 (1999).

[10] R. Dieckmann, Z. Phys. Chem. 107, 189 (1977).

[11] M. G. Lagally, D. E. Savage, and M.C. Tringides, in $R e-$ flection High-Energy Electron Diffraction and Reflection Electron Imaging of Surfaces, edited by P. K. Larsen and P. J. Dobson (Plenum Press, New York, 1987), p. 139.

[12] M. J. Carey et al., J. Mater. Res. 6, 2680 (1991).

[13] For a review, see W. Kleemann, Int. J. Mod. Phys. B 7, 2469 (1993); D. P. Belanger in Spin Glasses and Random Fields, edited by A. P. Young (World Scientific, Singapore, 1998).

[14] U. Nowak and K. D. Usadel, Phys. Rev. B 46, 8329 (1992); J. Esser, U. Nowak, and K. D. Usadel, Phys. Rev. B 55, 5866 (1997).

[15] T. J. Moran, J. M. Gallego, and Ivan K. Schuller, J. Appl. Phys. 78, 1887 (1995).

[16] J. Nogués et al., Phys. Rev. B 59, 6984 (1999).

[17] R. P. Michel, A. Chaiken, and C. T. Wang, J. Appl. Phys. 81, 5374 (1997).

[18] J. Nogués et al., Phys. Rev. Lett. 76, 4624 (1996).

[19] T. J. Moran and Ivan K. Schuller, J. Appl. Phys. 79, 5109 (1996).

[20] P. A. A. van der Heijden et al., Appl. Phys. Lett. 72, 492 (1998); J. Appl. Phys. 83, 7207 (1998).

[21] S-J. Han et al., Phys. Rev. B 45, 9728 (1992).

[22] U. Nowak, J. Esser, and K. D. Usadel, Physica (Amsterdam) 323A, 40 (1996); M. Staats, U. Nowak, and K. D. Usadel, Phase Transit. 65, 159 (1998). 